\title{
Microstructural characterization of alnico 9 alloy
}

Lin Zhou ${ }^{1}$, M. Miller ${ }^{2}$, D. A. Cullen ${ }^{2}$, Ping Lu ${ }^{3}$, R. W. McCallum ${ }^{1}$, I. E. Anderson ${ }^{1}$, S. Constantinides ${ }^{4}$, M. J. Kramer ${ }^{1}$

${ }^{1}$ Ames Lab, Ames, IA 50011

${ }^{2}$ Oak Ridge National Laboratory, Oak Ridge, TN 37831

${ }^{3}$ Sandia National Laboratories, PO Box 5800, MS 1411, Albuquerque, NM 87185

${ }^{4}$ Arnold Magnetic Technologies Corp., 770 Linden Avenue, Rochester, NY 14625

Permanent magnets (PM) with rear-earth (RE) elements, such as dysprosium (Dy) and neodymium $(\mathrm{Nd})$, have been widely used in motors and generators for hybrid electronic vehicles and wind turbines.[1] Concern over supply and price of the RE alloys has stimulated the search for alternative PMs.[1,2] One of the attractive non-RE PMs is alnico, a family of magnetic alloys composed primarily of $\mathrm{Al}, \mathrm{Ni}, \mathrm{Co}$ and $\mathrm{Fe}$, with excellent magnetic stability at high temperature. The magnetic properties of alnico alloys are closely related to the control of the spinodal decomposition (SD) into an FeCo-rich ( $\alpha_{1}$ phase) hard magnetic phase and a non-magnetic NiAlrich phase ( $\alpha_{2}$ phase). Improving alnico will require subtle changes in chemistry and processing to reduce the diameter of the magnetic phase while maintaining its volume fraction. Alnico 9 is the current available commercial alnico alloy with the highest energy product $(\mathrm{BH})_{\max }$, which is both grain aligned and spinodally decomposed with an applied magnetic field.[1] Needed improvements can only be achieved through a better understanding of nanostructuring during SD. This study focuses on structural characterization of alnico 9 alloy from Arnold Magnetic Technologies. Atom-probe tomography (APT) and a combination of TEM techniques, including diffraction contrast TEM, high resolution transmission electron microscopy (HREM), high-angle annular-dark-field (HAADF) scanning transmission electron microscopy (STEM), energy dispersive X-ray spectroscopy, and Lorentz microscopy, were used.

The interpenetrating nature of the $\alpha_{1}$ and $\alpha_{2}$ phase of alnico 9 is shown in Fig. 1 . The $\alpha_{2}$ phase is continuous, but whether the $\alpha_{1}$ phase consists offully isolated particles or has some degree of interconnectivity cannot be established definitively, because the volume sampled by the APT is too small relative to the size of the $\alpha_{1}$ phase. Fine $\mathrm{Cu}$-enriched rod-shaped particles with either a cylindrical or elliptical cross section were also observed at the corners of adjacent cuboidal $\alpha_{1}$ phase, and they occupy $\sim 4 \%$ volume fraction of the alloy. Moreover, a Ni-enriched rod-shape phase parallel to the primary $\alpha_{1} / \alpha_{2}$ interface in the NiAlTi phase was detected.

A nanometer scale mosaic structure formed by SD in alnico 9 (viewed along the transverse direction) was shown in Fig. 2 (a). The red bricks with a size of $\sim 35 \mathrm{~nm}$ are $\alpha_{1}$ precipitates, while the dark blue mold with similar size is $\alpha_{2}$ matrix. The $\alpha_{1} / \alpha_{2}$ interface facets along $\{110\}$ planes as well as $\{100\}$ planes. Cu rods (light blue, $\sim 5 \mathrm{~nm}$ ) sit in the $\alpha_{2}$ phase at the corner of two $\{110\} \alpha_{1}$ facets. A Ni-rich (green) shell was observed in the $\alpha_{2}$ phase at the $\alpha_{1} / \alpha_{2}$ boundary. Ti partitioned to the $\alpha_{2}$ phase. Selected area diffraction pattern taken along [110] zone axis confirms the $\alpha_{2}-$ phase has a L2 1 ordered structure. Aberration-corrected STEM images of the Cu-rich phase indicates that when the Cu-rich phase has a small diameter (cylindrical shape, Fig. 2 (b)), it is forms a coherent interface with the $\alpha_{2}$-phase. For $\mathrm{Cu}$-enriched phase with larger diameter, either intrinsic or extrinsic stacking faults were introduced. The $\mathrm{Cu}$-rich phase forms an elliptical rod shape with distorted fcc structure (Fig. 2(c)). Further, Ni-enriched particles were observed in the 
$\alpha_{2}$ phase when the $\alpha_{1}$ phase is faceted on $\{100\}$ planes. Observation of alnico 9 along the longitudinal direction (Fig. 2(d)) showed that the $\alpha_{l}$ precipitates were very long $(>400 \mathrm{~nm})$ and generally had tapered ends with an aspect ratio $>10$. Branching was commonly observed in the $\alpha_{1}$ rods. An in situ Lorentz microscopy study was also performed to analyze the movement of magnetic domains with an external magnetic field.

Research was supported by U.S. DOE, Office of Energy Efficiency and Renewable Energy (EERE), under its Vehicle Technologies Program, through the Ames Laboratory, Iowa State University under contract DE-AC02-07CH11358. Atom-probe tomography research (MKM) and aberration corrected TEM imaging was supported through a user project supported by ORNL's Center for Nanophase Materials Sciences (CNMS), which is a DOE Office of Science User Facility.

[1] M. J. Kramer, R. W. McCallum, I. A. Anderson, and S. Constantinides, JOM , 64, 752, (2012).

[2] Lin Zhou , M. K. Miller, Ping Lu, Liqin Ke, R. Skomski, M. McCartney, D. Smith, H. Dillon, Q. Xing, A. Palasyuk, S. Constantinides, R.W. McCallum, I. E. Anderson, V. Antropov, and M. J. Krame, Acta Materilia, 74, (2014), 224.

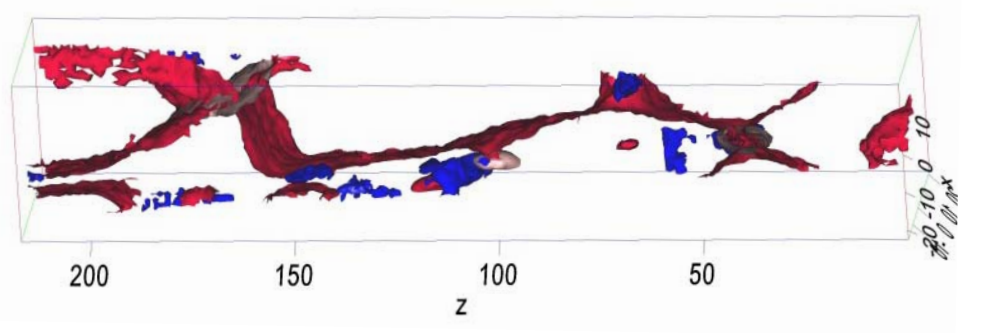

Figure 1. Isoconcentration surfaces: $10 \% \mathrm{Cu}$ to show the $\mathrm{Cu}$ (brown), $10 \% \mathrm{Ni}$ to show the outline of the NiAlTi phase/FeCo phases (red) and 30\% Ni to show the high Ni regions (blue). $\mathrm{Cu}$-enriched rods are at the corners of the FeCo phase and the $\mathrm{NiAl}$ region is contiguous.
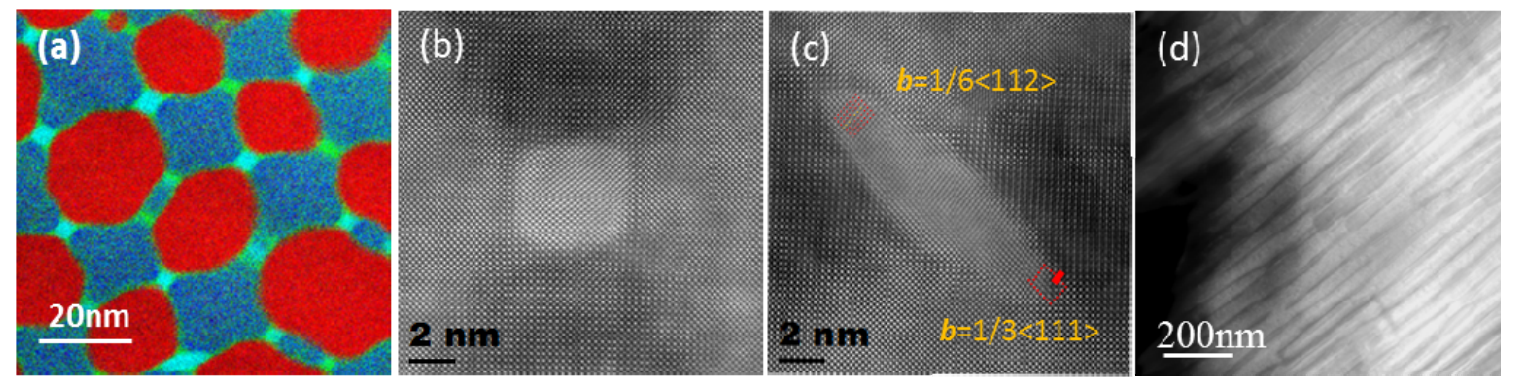

Figure 2. (a) Color composite energy-dispersive-X-ray map of alnico 9 taken along [001] crystal direction; (b,c) aberration corrected HAADF STEM images of alnico 9 taken under [100] zone axis along transverse direction. (d) HAADF STEM image of alnico 9 along longitudinal direction. 